\title{
EFEITOS DO BROMAZEPAM OBSERVADOS PELA ELETROENCEFALOGRAFIA QUANTITATIVA (EEGq) DURANTE A PRÁTICA DE DATILOGRAFIA
}

\author{
Dionis Machado', Victor Hugo Bastos², Marlo Cunha³, Vernon Furtado4, \\ Maurício Cagy ${ }^{5}$, Roberto Piedade ${ }^{6}$, Pedro Ribeiro ${ }^{7}$
}

\begin{abstract}
RESUMO - A eficiência com que uma informação é transmitida dentro da circuitaria neural pode ser alterada por neuromoduladores. $\mathrm{O}$ uso do Bromazepam nos transtornos de ansiedade se deve a sua propriedade ansiolítica. Porém, os efeitos deste benzodiazepínico na aprendizagem motora não são plenamente conhecidos. 0 objetivo deste estudo foi analisar alterações neuropsicológicas, comportamentais e eletrofisiológicas decorrentes da administração de Bromazepam $(6 \mathrm{mg}$ ) durante o aprendizado de uma tarefa motora. A amostra consistiu de 26 sujeitos saudáveis, de ambos os sexos, entre 19 e 36 anos. Os grupos controle (placebo) e experimental (Bromazepam $6 \mathrm{mg}$ ) foram submetidos ao aprendizado de datilografia, em desenho duplo-cego randomizado. Os resultados não revelaram diferenças nas variáveis neuropsicológicas e comportamentais entre os grupos. Testes estatísticos demonstraram interação entre condição e momento e um efeito principal para setor, ou seja, uma diminuição da potência relativa no hemisfério direito. Esta diminuição de potência sugere uma especialização da circuitaria neural no hemisfério contralateral ao dedo utilizado no pressionamento da tecla. Tal diminuição é independente do uso da droga.
\end{abstract}

PALAVRAS-CHAVE: Bromazepam, EEGq, aprendizagem motora.

\begin{abstract}
Effects of Bromazepam in qEEG by typingwriting
ABSTRACT - The efficiency with which an information is processed by the brain's neural circuitry can be altered by neuromodulators. The use of Bromazepam in the pharmacological treatment of anxiety disorders is due to its anxiolytic property. However, the effects of this benzodiazepine in motor learning tasks are not entirely understood. In this context, the goal of this study was to assess the effects of Bromazepam $(6 \mathrm{mg})$ on psychophysiological, behavioral, and electrophysiological variables, during the process of learning a motor task. The sample consisted of 26 healthy individuals, of both sexes, between 19 and 36 years of age. The control (placebo) and experimental (Bromazepam $6 \mathrm{mg}$ ) groups were submitted to a typewriting task, in a randomized, doble-blind design. The results did not reveal differences for phychophysiological and behavioral variables between the groups. Statistical tests pointed out to an interaction between condition and moment, and a hemisphere main effect, i.e. a reduction of relative power in the right hemisphere. This reduction suggests a specialization of the neural circuitry in the hemisphere contralateral to the finger used in the task. Such reduction is independent from the drug administration.
\end{abstract}

KEY WORDS: Bromazepam, qEEG, motor learning.

Desde o nascimento, o ser humano vivencia p rocessos diversificados de aprendizagem. Inicialmente, ele aprende a andar e a falar, com o decorrer da vida, vai sendo submetido a aprendizagens cada vez mais elaboradas ${ }^{1}$. Em termos gerais, pode ser dito que o indivíduo é predisposto a duas naturezas de aprendizagem: intelectual (referente ao pensamento) e motora (que diz respeito aos gestos corporais). Entretanto, pensamentos e movimentos se integram constantemente na realização das intenções do sujeito, seja quando uma idéia necessita ser executada ou quando um gesto precisa ser planejado ${ }^{2}$. No or-

\footnotetext{
${ }^{1}$ Mestranda, Laboratório de Mapeamento Cerebral e Integração Sensório-Motora, Instituto de Psiquiatria, Universidade Federal do Rio de Janeiro, Rio de Janeiro RJ, Brasil / IPUB / UFRJ; ${ }^{2}$ Doutorando, Laboratório de Mapeamento Cerebral e Integração SensórioMotora / IPUB / UFRJ; ${ }^{3}$ Mestrando, Laboratório de Mapeamento Cerebral e Integração Sensório-Motora / IPUB / UFRJ; ${ }^{4 P r o f e s s o r}$ Titular, Universidade Castelo Branco; ${ }^{5} \mathrm{D}$.Sc. em Engenharia Biomédica, COPPE, UFRJ; ${ }^{6} \mathrm{P}$ rofessor Adjunto III Doutor, IPUB, Coord en ad or do Laboratório de Mapeamento Cerebral e Integração Sensório-Motora, UFRJ; ${ }^{7}$ Professor Adjunto II PhD, Escola de Educação Física e Desportos, IPUB, Laboratório de Mapeamento Cerebral e Integração Sensório-Motora, UFRJ; Professor Pesquisador, Universidade Castelo Branco.
}

Recebido 23 Agosto 2004, recebido na forma final 27 Novembro 2004. Aceito 29 Janeiro 2005.

Dr. Victor Hugo do Vale Bastos - Rua Silva Teles 30A/208 - 20541-110 Andaraí RJ - Brasil. E-mail: victorhvbastos@uol.com.br 
ganismo, a ocorrência de tais processos é viabilizada por circuitos neurais complexos. A comunicação destes circuitos entre si é realizada através de neurotransmissores que em essência são responsáveis pelo processamento de informações. Os processos de neurotransmissão no sistema nervoso são modulados por uma classe de substâncias conhecidas como neuromoduladores ${ }^{3}$. Estes têm a propriedade de facilitar ou deprimir a ação de um devido neurotransmissor, dada a respectiva natureza. Isso se reflete na eficiência com que uma informação é trans mitida dentro do circuito neural. Atualmente, muitos distúrbios comportamentais são tratados através de drogas que atuam como neuromoduladores. Especificamente, a administração de compostos benzodiazepínicos auxilia no tratamento de distúrbios como ansiedade, insônia e tensão nervosa.

Os benzodiazepínicos eram concebidos como moléculas artificiais sintetizadas em laboratório, entretanto, verdadeiros receptores dessas substâncias foram observados na membrana de neurônios cerebrais ${ }^{4}$. A partir destes dados houve uma procura de substâncias endógenas que constituiriam os ligantes desses recepto res. Há maior probabilidade de que essas sejam provenientes da dieta ao invés de fabricadas pelo organismo. A ação dos benzodiazepínicos (representados aqui pelo Bromazepam) ao nível molecular é neuromodulatória, aumentando a afinidade dos receptores de ácido gama aminobutírico (GABA) pelo seu neurotransmissor ${ }^{5}$.

O Bromazepam, derivado benzodiazepínico tem a seguinte designação: 7-bromo-1, 3-dihydro 5-(2-pyridyl)-2H-1, 4-benzodiazepínico-2. Tem sido amplamente utilizado na Psiquiatria há cerca de 4 décadas para tratamento dos transtornos de ansiedade por apresentar propriedades ansiolítica, anticonvulsiva, miorelaxante e hipnótica em caso de dose elevada ${ }^{6-8}$. Os efeitos ansiolítico, analgésico e hipnótico do Bromazepam são exercidos pela intensificação da neurotransmissão mediada pelo GABA. Embora sua propriedade ansiolítica seja reconhecida como benefício nos transtornos de ansiedade, os efeitos das demais propriedades são questionáveis principalmente no que diz respeito à execução de tarefas motoras. Muitos estudos investigam os efeitos de benzodiazepínicos em distúrbios do humor e contrapartida, poucos experimentos relacionam tais efeitos a tarefas que envolvam integração sensório-motora. Segundo estes últimos, - Bromazepam parece causar certo grau de incoordenação motora, perturbação da marcha e há relatos de que ocorre aumento do tempo de reação (TR) e déficit de memória imediata em função de seu uso ${ }^{6,7,9,10}$. O presente estudo foi elaborado para contribuir com as pesquisas relacionadas à atuação do Bromazepam na execução de tarefas motoras. A utilização da eletroencefalografia quantitativa (EEGq) favorece tal estudo visto ser um método sensível para mensuração dos efeitos farma cológicos no Sistema Nervoso Central ${ }^{11}$. O objetivo é avaliar alterações neuropsicológicas, comportamentais e eletrofisiológicas decorrentes da administração de $6 \mathrm{mg}$ de Bromazepam comparando ao grupo placebo mediante a tarefa de datilografia.

\section{MÉTODO}

Amostra - Foi constituída por 30 estudantes de Graduação e Pós-Graduação dos cursos na área de saúde. Como critérios de inclusão foram considerados os seguintes aspectos: ausência de comprometimento da saúde física e mental (anamnese prévia); não serem usuários de substâncias psicotrópicas ou psicoativas; sono de 6 a 8 horas na noite anterior ao experimento; inexperiência prévia em datilografia, dominância manual destra de a cordo com o inventário de Edinburgh ${ }^{12}$. Todos os sujeitos tinham se alimentado moderadamente até duas horas antes do experimento. Quatro sujeitos foram descartados, pois embora tenham relatado inexperiência na tarefa de datilografia apresentaram as variáveis comportamentais (tempo e erro) compatíveis com a de sujeitos experientes (menos de $5 \%$ de erro e/ou tempo inferior a 5 minutos no primeiro bloco da tarefa). Os erros foram considerados conforme critérios pré-estabelecidos e conferidos por três dos autores.

Os sujeitos foram informados a respeito do objetivo do estudo e assinaram declaração de consentimento na qual a condição experimental encontrava-se detalhadamente descrita. $\mathrm{O}$ experimento foi aprovado pela Comissão de Ética do Instituto de Psiquiatria da Universidade Federal do Rio de Janeiro.

Desenho experimental - Ao chegar no laboratório no horário previamente agendado, o sujeito era informado quanto ao procedimento experimental. Após concordar e assinar a declaração de consentimento tinha início a primeira captação de sinal por meio do eletroencefalograma (EEG). Esta primeira captação consistia em seis minutos nos quais o sujeito mantinha-se de olhos fechados e seis minutos de olhos abertos, sendo solicitado evitar piscadas a fim de minimizar a quantidade de artefatos musculares no EEG. Após esta primeira etapa de captação o sujeito ingeria uma cápsula que poderia conter placebo (amido 400mg) ou 6mg de Bromazepam. A distribuição das cápsulas seguiu um modelo duplo-cego para evitar que houvesse influência nos resultados por parte dos examinadores. Trinta minutos após a ingestão da cápsula era feita avaliação do nível de atenção do sujeito pelo teste de Stroop. Tal teste também tem sido empregado em alguns estudos para promover ansie- 
dade ${ }^{13,14}$. Após o teste era iniciada nova captação, desta vez simultaneamente à tarefa da datilografia. Ao término da tarefa era realizada mais uma etapa de captação (seis minutos com olhos fechados e seis minutos com olhos abertos).

Aquisição de dados - Para a captação do sinal eletroencefalográfico foi utilizado o aparelho Braintech 3000 (EMSA - Instrumentos Médicos, Brasil), sistema que utiliza uma placa conversora analógica-digital (A/D) de 32 canais com resolução de 12 bits, colocada em slote ISA de Pentium III - com processador de $750 \mathrm{~Hz}$. Foi utilizada uma touca que continha os eletrodos dispostos de acordo com o sistema internacional $10-20^{15}$, incluindo-se os elet rodos de referência posicionados nos lóbulos das orelhas (bi-auricular) ${ }^{16}$. O tamanho da touca utilizada estava de a cordo com o perímetro craniano de cada sujeito (toucas de tamanhos variados). A sala utilizada para captação do sinal eletroencefalográfico foi preparada com isolamento acústico e elétrico; durante a aquisição do sinal as luzes foram reduzidas, exceção feita ao momento da tarefa.

O sinal adquirido em determinado eletrodo foi resultante da diferença entre o potencial elétrico do mesmo no escalpo e a referência pré-estabelecida. Foram verificados, "a priori", os níveis de impedância de cada elet rodo, cujos valores foram fixados entre 5-10K ohms $(\theta)$ e mantidos nesses padrões. Os sinais adquiridos deviam estar com o total de amplitude (pico a pico) menor que $100 \mu \mathrm{V}$. Por este motivo, o sinal deveria ser amplificado com ganhos variando de 20000 a 50000 . Os sinais eletroencefalográficos adquiridos deveriam flutuar entre 0,01 e $50 \mathrm{~Hz}$. A atividade elétrica ocular foi estimada com a colocação de dois eletrodos de $9 \mathrm{~mm}$ de diâmetro montados de forma bipolar. Os eletrodos foram posicionados, respectivamente, acima e abaixo da órbita do olho dire ito para registrar movimentos oculares verticais e no canto externo(Canthus) do mesmo para registrar movimentos oculares horizontais.

Os parâmetros do EEG quantitativo foram extraídos de trechos de 2 segundos antes e depois do início do movimento em 20 derivações de acordo com o sistema 1020 de posicionamento de eletrodos. Trechos de sinal contaminados por artefatos musculares foram retirados em duas fases: os trechos aceitos por um algoritmo de rejeição automática de artefatos por trespasse de limiar (100 $\mu \mathrm{V})$ passaram por inspeção visual para garantir uma seleção mais criteriosa de trechos válidos. A partir da estimativa da densidade espectral de potência, obteve-se a potência nas bandas do EEG (delta, teta, alfa e beta) antes do início do movimento (entre -2 s e $0 \mathrm{~s}$, considerado como referência - R) e após o movimento (entre 0 s e 2 $S$, denominado $P$ ), com relação a cada pressionamento de tecla de interesse. Em 0 s tem-se a representação do momento exato do pressionamento da tecla de interesse. Além da potência em cada banda, a potência total também foi estimada visando à obtenção da potência relativa, definida como a razão entre a potência em cada banda e a potência total. Com o objetivo de facilitar a visualização, todos trechos de sinal contidos entre $2 \mathrm{~s}$ antes e 2 s após o pressionamento da tecla de interesse sofreram filtragem passa-faixa (associação de filtros passa-altas e passa-baixas Butterworth de ordem 2 cada). Uma vez filtrados os respectivos trechos de dados, os mesmos foram elevados ao quadrado e sofreram filtragem passa-baixas objetivando a detecção de envoltória. Uma promediação de todas as trilhas ocorreu para se obter à evolução temporal da potência média em todas as 20 derivações utilizadas, que pôde ser interpretado visualmente e servir de suporte às conclusões estatísticas.

Procedimento da tarefa motora - Para a realização da tarefa optou-se por máquina de datilografia de modelo antigo (Olivetti Línea 98), tal opção se deve ao fato da representação cortical ser maior quanto maior o grau de força empregada na tarefa ${ }^{17}$. Os sujeitos estavam confortavelmente sentados em uma cadeira com suporte para os antebraços a fim de minimizar os artefatos musculares. A distância entre a cadeira do sujeito e a mesa onde se encontrava a máquina de datilografia foi de c e rca de $20 \mathrm{~cm}$ variando em função do comprimento do antebraço de cada sujeito. O teclado da máquina foi coberto com uma caixa de madeira que evitava a visualização do posicionamento das mãos sobre o teclado e que exigia do sujeito a criação de uma "referência espacial" para o teclado.

A tarefa consistiu num exercício de datilografia com a p rendizagem prog ressiva e realizada num único dia. Tal exercício era composto por quatro blocos sendo cada bloco constituído por doze linhas. Cada linha deveria conter cinco seqüências de letras para cada mão. A seqüência de letras para cada mão era assim estabelecida: asdfg para mão esquerda e çlkjh para mão direita. A seqüência da mão esquerda era iniciada pelo dedo mínimo pressionando a tecla referente à letra $a$, em seguida ocorria o p ressionamento da tecla s pelo anular, tecla $d$ pelo dedo médio, tecla $f$ e $g$ pelo indicador. Te rminada a seqüência da mão esquerda era efetuado o pressionamento da tecla espaço, utilizando para isto o polegar esquerdo ou o direito, e então era iniciada a seqüência da mão direita (dedo mínimo pressionando ç, anular na tecla $l$, médio para $k$, indicador para as teclas $j$ e $h$ ).

Abaixo do teclado da máquina de datilografia existia um circuito elétrico responsável pela captação do pressionamento das teclas de interesse, a saber, a e $g$, pois respectivamente representavam início e término da seqüência da mão esquerda, ç e $h$ pelo mesmo motivo em função da mão direita e a tecla espaço que representava mudança para seqüência seguinte. Tal circuito era composto por cinco dispositivos em forma de " $U$ " denominados optoeletrônicos. Cada optoeletrônico possui uma porção emissora e uma porção receptora de luz que permitia a transferência da informação de pressionamento das teclas de interesse para o sistema de aquisição de dados onde eram efetivamente reconhecidas as letras con- 
forme seus traçados gráficos característicos (mediante passagem por um simples conversor digital-analógico).

Bandas de freqüência e localização espacial - Os dados eletrofisiológicos de interesse referem-se à banda teta. A escolha pela banda teta justifica-se pelo fato desta estar relacionada a mecanismos de potencialização de longa duração e depressão de longa duração que fundamentam bioquimicamente o aprendizado. Manipulações experimentais que suprimem as oscilações de teta tendem a causar prejuízo no aprendizado, ao passo que drogas conhecidas por aumentar as funções de memória em ratos evidenciam aumento na amplitude teta. Amplitude, freqüência e fase da banda teta mostram-se críticas para o processo de aprendizagem ${ }^{16,18}$.

Foram selecionados os eletrodos localizados em áreas frontais e parietais. Áreas frontais foram selecionadas em virtude da relação destas aos mecanismos de motivação, planejamento e execução de movimentos voluntários. A inserção dos eletrodos parietais justifica-se pelo controle dos mecanismos sensoriais e espaciais ocorrerem em tais áreas ${ }^{19}$. Esta seleção objetiva comparar os achados eletrofisiológicos entre os dois hemisférios cerebrais. Desta fo rma foram selecionados os seguintes eletrodos frontoparietais: FP1, FP2, F3, F4, F7, F8, C3, C4, P3, P4.

Análise estatística - Em relação ao teste de Stroop foi realizada um teste-t (grupos independentes) para tempo e escore bruto. A realização de uma única testagem ( S t roop) está em função do efeito aprendizado do teste. Particularmente, este teste tem sido utilizado para analise dos processos de atenção em modelos com eletroencefalografia 20 .

Para análise comportamental, tempo de execução e erros cometidos na tarefa, foi feito o teste de ANOVA (Two-Way). Em especial, ANOVA foi implementada para comparar dois fatores: condição (placebo e Bromazepam $6 \mathrm{mg}$ ) e bloco (bloco I e bloco III). Estes blocos foram selecionados visto que o primeiro indica o momento de inexperiência do indivíduo e o terceiro indica um momento do aprendizado. O segundo bloco não foi selecionado já que o tempo era curto para um aprendizado tão efetivo como provavelmente ocorreria no terceiro bloco. $\mathrm{O}$ quarto bloco também não foi selecionado neste modelo já que parece ser o bloco em que o sujeito apresenta um certo grau de fadiga em relação à tarefa.

A análise dos dados eletrofisiológicos considerou a tarefa completa e não a subdivisão em blocos considerada na análise comportamental. Os valores de potência relativa obtidos (dados eletrofisiológicos) 2 segundos antes do pressionamento da tecla $\underline{a}$, e 2 segundos após o pressionamento, foram transformados para logaritmo natural utilizando o softwa reSPSS versão 10.0. Esta transform ação se fez necessária porque os valores de potência relativa não apresentam uma distribuição Gaussiana. Testes de homocedasticidade demonstraram que os dados em logaritmo natural apresentam uma distribuição normal ${ }^{21}$. Em relação aos dados eletrofisiológicos, o teste de ANOVA
(Three-Way) comparou os fatores: condição (controle $x$ experimental); momento (dois segundos antes pressionamento da tecla $\underline{a}$, e dois segundos após do pressionamento da tecla) e setores no escalpo (hemisfério direito - FP1, F3, C3, F7, P3 e esquerdo - FP2, F4, C4, F8, P4). Para todas as análises foi considerado um $p$ estatístico menor ou igual a $0,05(p \leq 0,050)$.

\section{RESULTADOS}

Os resultados descritos a seguir foram divididos em três formas de variáveis dependentes: neuro $p$ sicológica, comportamentais e eletrofisiológicas. A neuropsicológica voltada para o nível de atenção, as comportamentais para a caracterização da perf o rmance, e as eletrofisiológicas para as alterações plásticas neurocorticais.

Variável neuropsicológica - O teste utilizado para verificar nível de atenção (teste de Stroop) não mostrou diferença significativa entre o grupo placebo e o experimental tanto para a variável tempo de execução do teste $(p=0,976)$, quanto para esco rebruto atingido pelos participantes $(p=0,332)$.

Variáveis comportamentais - Os resultados do teste de ANOVA (Two-Way), condições (Placebo e Experimental) versus blocos (Bloco I e Bloco III), na variável tempo de execução da tarefa (Fig.1) apresentou um efeito principal para blocos $(p=0,001)$. Em especial, não houve interação entre condição versus blocos, e não foi demonstrado efeito principal entre as condições. A ANOVA (Two-Way), condições e blocos, no número de erros (Fig.2), apresentou um efeito principal entre blocos $(p=0,011)$. Não foram observadas interações entre condição versus blocos, e não houve diferença significativa entre as condições.

Variáveis eletrofisiológicas - Nas análises eletrofisiológicas foram considerados os valores de potência relativa na banda teta, dois segundos antes do pressionamento da tecla a e dois segundos depois do pressionamento (Fig 3). Os resultados da ANOVA ( Th ree-way), ou seja, condição, momento e setore $s$ no escalpo não mostraram uma significativa interação $\left(F_{(2,517)}=0,015 \mathrm{p}=0,901\right)$. Não foi observada interação entre momento versus setor no escalpo $\left(F_{(2,517)}=0,528 \mathrm{p}=0,468\right)$, e não houve interação e n tre condição versus setor $\left(F_{(2,517)}=2,678 p=0,102\right)$. Em contrapartida, ocorreu uma interação significativa entre condição versus momento $\left(F_{(2,517)}=16,445 \mathrm{p}\right.$ 0). Uma vez observada a interação entre condição versus momento os respectivos efeitos principais foram ignorados (condição e momento). Sendo 


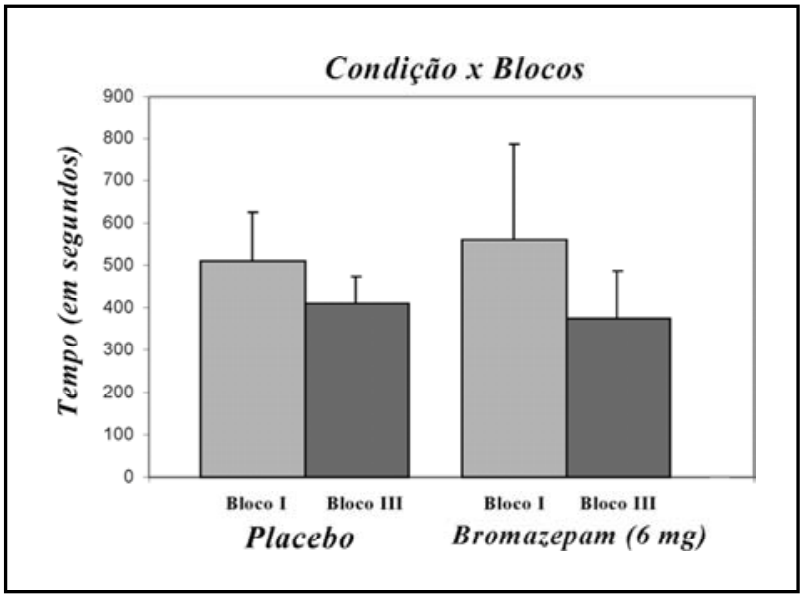

Fig 1. Relação entre tempo de execução da tarefa nos blocos I e III nas diferentes condições (controle e experimental).

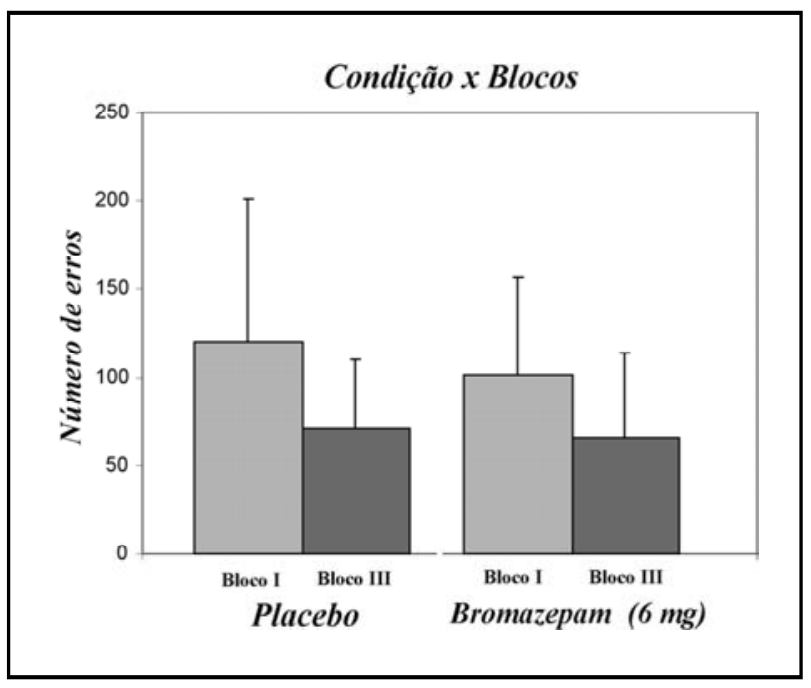

Fig 2. Relação entre os erros dos blocos I e III nos grupos con trole e experimental.

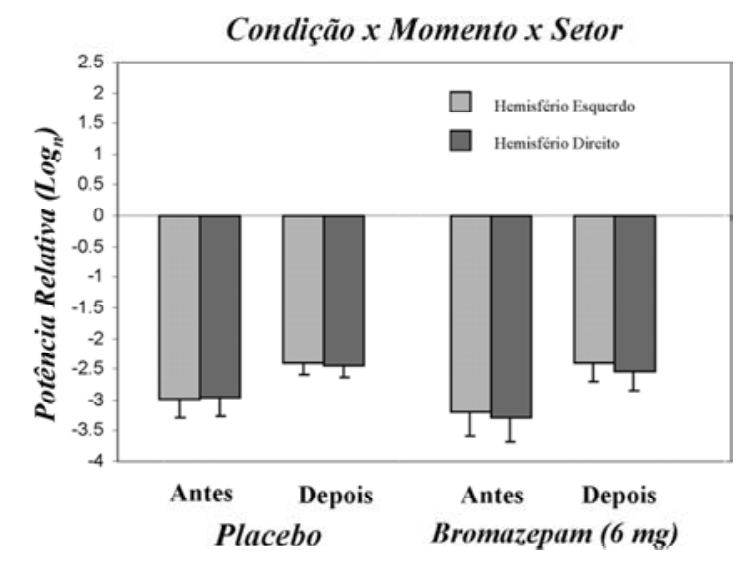

Fig 3. Relação entre os valores de potência relativa na banda teta considerando condição $x$ momento $x$ setor. assim, destaca-se o efeito principal somente no fator setor $\left(F_{(2,517)}=5,417 \mathrm{p}=0,020\right)$.

\section{DISCUSSÃO}

O objetivo do presente experimento foi obsenvar alterações neuropsicológicas, comportamentais e eletrofisiológicas em função da administração de $\mathrm{B}$ romazepam em indivíduos expostos a uma tare fa motora. Em particular, o Bromazepam é um benzodiazepínico e atua como agonista dos receptores do GABA. Tais receptores são classificados em GABA $A$ e GABA B. GABA A é ionotrópico e abre canais de Cloreto ao passo que GABA $B$ é metabolotrópico e abre os canais de Potássio. Ambos promovem uma hiperpolarização da membrana neural que pode ser intensificada com o uso desta substância 5 .

Variável neuropsicológica - Os resultados do teste de Stroop empregado em nosso modelo experimental não demonstraram diferenças entre os grupos (Placebo e Bromazepam 6mg). Especificamente, tais diferenças não foram percebidas em relação ao escore bruto, ou mesmo em relação ao tempo para execução do teste. De acordo com estes achados nos parece que a droga não gerou alterações nos níveis de atenção capazes de serem detectadas pelo teste. Em outros modelos o teste parece ser uma ferramenta de valor para medidas cognitivas. Alguns modelos de estudos utilizaram o teste de Stroop na tentativa de provocar a ansiedade e nesta situação foram avaliados os efeitos ansiolíticos do Diazepam em diferentes doses (5 e $10 \mathrm{mg}$ ). Tais efeitos foram comparados entre homens e mulheres. Foi verificado que nas mulheres não houve resposta apesar do medicamento ${ }^{14}$. As aplicações do teste de Stroop e de suas variações podem ser utilizadas em diversos modelos com as variáveis extraídas de medidas eletroencefalográficas, como por exemplo o potencial evocado visual e os eventos relacionados à tarefa ${ }^{22}$. Vários autores tentar explicar o chamado "efeito Stropp" sob várias metodologias além de mostrar que este teste pode ser utilizado para verificar algumas modalidades cognitivas de maneira associada a variável coerência do eletroencefalograma ${ }^{20}$.

Variáveis comportamentais - Duas variáveis comportamentais (tempo e erro) foram usadas para mensurar processos de incorporação de memória de procedimentos durante o aprendizado de datilografia. Na relação entre condições versus blocos não se verificou interação. A condição também não mostrou relações estatisticamente significantes. $O$ efeito principal para blocos, encontrado em ambas 
variáveis (tempo e erros), parece estar relacionado ao efeito do aprendizado da tarefa e não ao uso da droga. Tais alterações nestas variáveis sugere $m$ a p rendizagem, pois evidenciam alterações plásticas decorrentes da fixação da tarefa motora do primeiro para o terceiro bloco ${ }^{23-25}$. É provável que o efeito ansiolítico da droga não tenha influenciado na performance motora, visto que na análise do fator condição (placebo versus Bromazepam de $6 \mathrm{mg}$ ) não se verificou diferenças estatísticas. $O$ simples fato de saber que está sendo testado gera no sujeito um sentimento de ansiedade. A instrução verbal dada no início da tarefa contribuiu neste sentido, pois criava no sujeito a responsabilidade de realizar a tarefa com o mínimo de erros e no menor tempo possível. Experimentos recentes demonstram que tarefas que envolvam processos cognitivos geram sensações de ansiedade ${ }^{14,26}$. Apesar da comprovada eficácia do Bromazepam (e outros benzodiazepínicos) sobre o controle da ansiedade, cientistas alertam sobre os possíveis inconvenientes a que seus usuários estão suscetíveis durante a rotina diária de trabalho ${ }^{9}$. Particularmente neste modelo, o efeito ansiolítico do medicamento parece não se mostrar relevante o suficiente a ponto de modificar as atividades corticais e ser captado pelo EEG.

Coincidindo com nossos achados, estudos submetendo indivíduos a testes de atenção, extensão de memória e TR utilizando Bromazepam em diferentes doses (1,5mg e $3 \mathrm{mg}$ ) e placebo não indicam prejuízo psicomotor tanto em função do medicamento quanto em função da dosagem. Ao contrário, com as doses administradas, houve um leve efeito positivo na performance que pode ser explicado pela diminuição do estado de ansiedade. No experimento de Hobi, os sujeitos foram submetidos a um consumo mais prolongado do medicamento. Os testes foram realizados ao longo de 14 dias. Em particular, houve um atraso no TR do terceiro grupo (3mg) em especial no sétimo dia, comparado aos outros grupos. Isso pode ser interpretado como decorrente de um efeito sedativo do Bromazepam. Quanto aos testes de atenção e alerta, ocorreram melhoras significativas nos três grupos (em função da aprendizagem). Isso sugere que em doses relativamente baixas, o Bromazepam não é capaz de influenciar substancialmente tais variáveis ${ }^{6}$, hipótese confirmada por dados pilotos de nosso laboratório. Investigações envolvendo vários benzodiazepínicos (alprazolam, bromazepam, diazepam e lorazepam) apontam diferentes resultados em função da dosagem e tempo de tratamento ${ }^{27}$.
Em contrapartida alguns estudos utilizando o potencial relacionado a eventos (PRE) em uma tarefa de vigilância visual relatam que o Bromazepam pode ter afetado a performance dos sujeitos ao nível global. Em tal experimento houve aumento da velocidade, porém comprometimento na precisão. O aumento da velocidade foi atribuído a redução de cautela provocada pelo medicamento. Conseqüentemente, o número de erros durante a tarefa aumentou. É sugerido pelo estudo do PRE, que o Bromazepam afete os estágios iniciais do processamento de informação (atenção/identificação) ${ }^{7}$. Tal achado é apoiado por outros autores que evidenciaram distúrbios de cognição e do processamento de dados sensoriais devidos ao Bromazepam ${ }^{10}$.

Variáveis eletrofisiológicas - Na avaliação das variáveis eletrofisiológicas foi selecionada a banda teta e os seguintes eletrodos: F8, P4, C4, F4, Fp2, F7, P3, C3, F4, Fp1. A banda de freqüência teta foi selecionada em função da associação desta com processos de automatismo, atenção versus aprendizagem $^{16,18}$. Os eletrodos selecionados representam o córtex frontal e somatosensorial, em especial com áreas representativas do controle motor bi-manual ${ }^{28}$. Os presentes achados não indicaram uma interação entre condição, momento e setor. Este resultado sugere que o uso do Bromazepam ( $6 \mathrm{mg}$ ) não produz alterações significativas na potência relativa em teta, aumento ou diminuição, nos 2 segundos que antecedem o pressionamento da tecla $\underline{a}$, ou 2 segundos após. Deve ser ressaltado que esta ausência de interação foi observada quando o córtex foi dividido entrehemisfério esquerdo e direito (setor).

Contrariamente, o presente experimento aponta para uma interação significativa entre condição e momento. Isto demonstra que tanto a droga quanto o momento influenciaram os eventos de sincronização e dessincronização nos parâmetros da potência relativa em teta. Uma análise mais detalhada demonstra que no grupo placebo ocorreu uma sincronização (aumento de potência) quando foram comparados os momentos antes e depois. O mesmo resultado foi constatado no grupo experimental. Tal sincronização pode ser considerada como uma desativação ou inibição de atividade em grupos neurais de áreas motoras. Os disparos neuronais tornam sincrônicos em virtude do processo de aprendizagem e ocorre implementação na comunicação entre tais grupos neuronais ${ }^{29}$. Estes achados experimentais são relevantes, pois coincidem com resultados de outros estudos que correlacionam a sincronização em teta a uma melhor performance 
de memória. Estes processos de sincronia em áreas corticais estão relacionados com outras variáveis cognitivas, tais como: aprendizagem espaço-temporal e memória de trabalho ${ }^{30}$. Além disso, alguns estudos demonstram aumento de potência em teta após a realização de tarefas que envolvam esforço mental e associam este achado à diminuição da vigilância ${ }^{26,30}$.

Os resultados deste experimento evidenciam efeitos principais nos fatores condição, momento e setor, isoladamente. Como foi verificada uma interação entre condição e momento não serão discutidos os efeitos principais dos dois fatores separadamente (condição e momento). Desta forma, como não houve interação entre setor e nem dos outros dois parâmetros, será discutido o efeito principal do hemisfério esquerdo e direito independentemente. Foram comparados os hemisférios direito e esquerdo para verificar as possíveis representações corticais determinadas em função da tarefa motora. Estes achados em relação ao efeito principal para setor são relevantes uma vez que o hemisfério direito teve sua potência relativa diminuída em relação ao hemisfério esquerdo. Esta diminuição da potência relativa em teta à direita pode ser correlacionada com as tendências de especialização n e u ronal induzidas pela tare $\mathrm{fa}$. Neste contexto, as áreas sensório-motoras do hemisfério direito contêm populações neuronais que codificam parâmetros do membro contra-lateral, mão esquerda.

Em conclusão, o presente estudo contribui com as pesquisas relacionadas à atuação do Bromazepam e execução de tarefas motoras. O uso de 6 miligramas de Bromazepam não promoveu prejuízo nesta tarefa motora. Nossos resultados coincidem com grande parte dos trabalhos relacionados à atividade teta e aprendizado. Sugere-se que o efeito do Bromazepam seja estudado através de tarefas que permitam expor o mesmo sujeito aos momentos placebo e droga. Além disso, poderia ser feito um acompanhamento em longo prazo verificando as alterações produzidas por um possível efeito cumulativo do medicamento. Outros trabaIhos devem ser desenvolvidos para se observar à dosagem específica para tais modelos.

\section{REFERÊNCIAS}

1. Ungerleider L, Doyon J, Karni, A. Imaging brain plasticity during motor skill learning. Neurobiology of learning and memory 2002;78:553-564.

2. Striedter G. A comparative perspective on motor learning. Neurobiology of learning and memory 1998;70:189-196.

3. Pascual-Leone A, Cammarota A, Wassermann E, Brasil-Neto J, Cohen L, Hallett M. Modulation of motor cortical outputs to the reading hand of braille readers. Ann Neurol 1993Jul;34:33-37.
4. Hegener O, Jordan R, Haberlein H. Benzodiazepine binding studies on living cells: application of small ligands for fluorescence correlation spectroscopy. Biol Chem 2002;383:1801-1807.

5. Oelschlager H. Chemical and pharmacologic aspects of benzodiazepines. Schweiz Rundsch Med Prax 1989;78:766-772.

6. Hobi V, Dubach UC, Skreta M, Forgo I, Riggenbach H. The subacute effect of Bromazepam on psychomotor activity and subjective mood. J Intern Med Res 1982;10:140-146.

7. Leeuwen Van TH, Verbaten MN, Koelega HS, Kenemans JL, Slangen JL. Effects of Bromazepam on single-trial event-related potentials in a visual vigilante task. Psychopharmacology 1992; 106:555-564.

8. Blanco C, Antia SX, Liebowitz MR. Pharmacotherapy of social anxiety disorder. Biol Psychiatry 2002;51:109-120.

9. Jansen A, Verbaten M, Slangen J. Acute effects of Bromazepam on signal detection performance, digit symbol substitution test and smooth pursuit eye movements. Neuropsychobiology 1988;20:91-95.

10. Bourin M, Auget JL, Colombel MC, Larousse C. Effects of single oral doses of Bromazepam, Buspirone and Clobazam on performance tasks and memory. Pharmacopsychiatry 1989;22:141-145.

11. Jahnig P, Jobert M. Methodological considerations for the evaluation of EEG mapping data: a practical example based on a placebo/diazepam crossover trial. Neuropsychobiology 1995;31:31-46.

12. Oldfield R. The assessment and analysis of handedness: the Edinburgh inventory. Neuropsycology 1971;9:97-113.

13. Mead LA, Mayer AR, Bobholz JA, Woodley SJ, Cunningham JM, Hammeke TA, Rao SM. Neural basis of the Stroop interference task: response competition or selective attention? J Int Neuropsychol Soc 2002;8:735-742.

14. Silva FT, Prado GB, Ribeiro LCG, Leite JR. The anxiogenic video-recorded Stroop color-word test: psychological and physiological alterations and effects of diazepam. Physiol Behav 2004;82:215-230.

15. Jasper $H$. The ten-twenty electrode system of the international federation. EEG Clin Neurophysiol 1958;10:371-375.

16. Niedermeyer E, Silva F. Electroencephalography: basic principles, clinical applications and related fields, 4.Ed. Baltimore: Urban \& Schwarzenberg, 1999:53-55.

17. Hamzei F, Dettmers C, Rzanny R, Liepert J, Büchel C, Weiller C. Reduction of excitability ("inhibition") in the ipsilateral primary motor cortex is mirro red by fMRI signal decreases. Neuroimage 2002; 17: 490-496.

18. Caplan JB, Madsen JR, Bonhage AS, Scheibe RA, Newman EL, Kahana MJ. Human $\theta$ oscilations related to sensorimotor integration and spatial learning. J Neurosc 2003;23:4726-4736.

19. Smyrnis N, Theleritis C, Evdokimidis I, Müri RM, Karandreas N. Single-pulse transcranial magnetic stimulation of parietal and prefrontal areas in a memory delay arm poiting task. J Neurophysiol 2003;89:3344-3350.

20. Schack B, Chen ACN, Mescha S, Witte H. Instantaneous EEG coherence analysis during the Stroop task. Clin Neurophysiol 1999;110:1410-1426.

21. Bucci P, Mucci A, Volpe U, Merlotti E, Galderisi S, Maj M. Executive hypercontrol in obsessive-compulsive disorder: electrophysiological and neuropsychological índices. Clin Neurophysiol 2004;115:1340-1348.

22. West R. Neural correlates of cognitive control and conflict detection in the Stroop and digit-location tasks. Neuropsychologia 2003;41:1122-1135.

23. Bastos VHV, Alves HVD, Piedade RAM, Silva VF, Silva APRS. Alterações corticais produzidas em função de uma tarefa de datilografia. Fit Perform 2002;1:53-58.

24. Bastos VHV, Veiga H, Cunha M, Guimarães MA, Piedade R, Ribeiro P. Assimetria inter-hemisférica em função da aprendizagem de uma tarefa de datilografia. Fisioterapia Brasil 2003;4:426-431.

25. Cunha M, Bastos VHV, Veiga H, Cagy M, Piedade R, Ribeiro P. Alterações na distribuição de potência cortical em função da consolidação da memória no aprendizado de datilografia. Arq Neuropsiquiatr 2004;62:662-668.

26. Smit AS, Eling PATM, Coenen AML. Mental effort affects vigilance enduringly: after-effects in EEG and behavior. Internat J Psychophysiol 2004;53:239-243.

27. Bourin M, Hascoet M, Mansouri B, Colombel MC, Bradwejn J. Comparison of behavioral effects after single and repeated administrations of four benzodiazepines in three mice behavioral models. J Psychiat Neurosci 1992;17:72-77.

28. Tecchio F, De Lucia M, Salustri C, et al. District-related frequency specificity in hand cortical representation: dynamics of regional activation and intra-regional synchronization. Brain Res 2004;1014:80-86.

29. Georgopouls AP, Kalaska JF, Caminiti R, Massey JT. On the relations between the direction of two-dimensional arm movements and cell discharge in primate motor cortex. J Neurosci 1982;2:1527-1537.

30. Jausovec N, Jausovec K. Differences in induced brain activity during the performance of learning and working-memory tasks related to intelligence. Brain Cogn 2004;54:65-74. 